\title{
THE PATHOLOGICAL EFFECTS PRODUCED BY SERA OF ANIMALS IMMUNIZED WITH FOREIGN NERVOUS OR SPLENIC TISSUE
}

\section{PART II : INTRA-ARTERIAL INJECTION OF SERUM}

\author{
BY
}

\section{E. WESTON HURST}

From Imperial Chemical (Pharmaceuticals) Limited, Hexagon House, Blackley, Manchester

In an earlier paper (Hurst, 1955) we have described the cerebral lesions which follow intracisternal injection in monkeys of the sera of goats immunized with simian brain, and have attributed them to an antigen-antibody reaction. The method of bringing the two components of the reaction into contact was, however, so " artificial " that it seemed improbable that it could throw light upon the genesis of lesions developing in animals immunized with nervous tissue, or of those obscure conditions in man in which sensitivity is believed to play a part. We therefore studied the effect of introducing the antisera into the blood-stream. Large amounts $(10 \mathrm{ml}$.) injected into the general circulation caused no apparent disturbance and, even when injected directly into the common carotid artery while blood was flowing through the vessel, the sera produced no detectable macroscopic or microscopic reaction in the brain. If, however, the latter procedure was followed after ligation of all branches of the common carotid artery other than the internal carotid, very marked disturbances ensued. As previously, we compared the effects produced by neurotoxic sera with those evoked by an anti-spleen serum, as well as, of course, the (negligible) effects of normal goat serum.

\section{Experimental}

Preparation of Sera and Operative Technique.-The sera were prepared in the manner described earlier (Hurst, 1955) and were stored at $-10^{\circ} \mathrm{C}$.

In monkeys anaesthetized with nembutal, we tied all branches of the right common carotid artery with the exception of the internal carotid. The sera, thawed and re-centrifuged immediately before use and then warmed to $37^{\circ} \mathrm{C}$., were injected slowly into the common carotid while blood flowed through the artery. The time taken for injection was two minutes for monkeys of the " $C$ " series (Table) and five to eight minutes for those of the " $H$ " series. After injection blood was allowed to flow through the artery for half-a-minute or so, before ligation of the vessel proximal to and distal to the needle puncture. If the animal survived it was sacrificed four to six days after operation.

A résumé of experiments performed appears in the Table.

\section{Effects of Injecting Neurotoxic Serum}

In many animals, during or immediately after the injection, there occurred more or less severe but transient movements of the diaphragm and abdominal wall, producing the effect of retching or of hiccough.

By the time the skin was sutured, or a few minutes later, the eyelids of the homolateral orbit began to swell ; two monkeys ( $\mathrm{H} 4$ and 7 ) receiving the smallest dose of serum, and one (H12) pre-treated with cortisone, provided the only exceptions to this statement. When death followed soon after injection, the brains of the animals showed the cerebral engorgement described below; thus the ocular manifestations, confined sharply to the area of distribution of the ophthalmic branch of the anterior cerebral artery, constituted outward and visible evidence of the reaction taking place within the cranium. With less severe reactions the eyelids were merely oedematous and the oedema disappeared within a day or so, usually leaving some residual difference in the palpebral fissures and in the size of the pupils due probably to disturbance of the sympathetic at operation. Other animals, however, showed " purple" oedema, with severe congestion and haemorrhages becoming more intense for many hours and persisting for several days or until death. The most extreme reaction occurred in $\mathrm{H10}$ and led to complete destruction of the eyeball, the dry and shrunken remnants of which six days later were surrounded by cicatrizing but still oedematous orbital and palpebral tissues. Immediately after the operation many animals lachrymated vigorously, usually from the homo- 
TABLE

RÉSUMÉ OF EXPERIMENTS PERFORMED

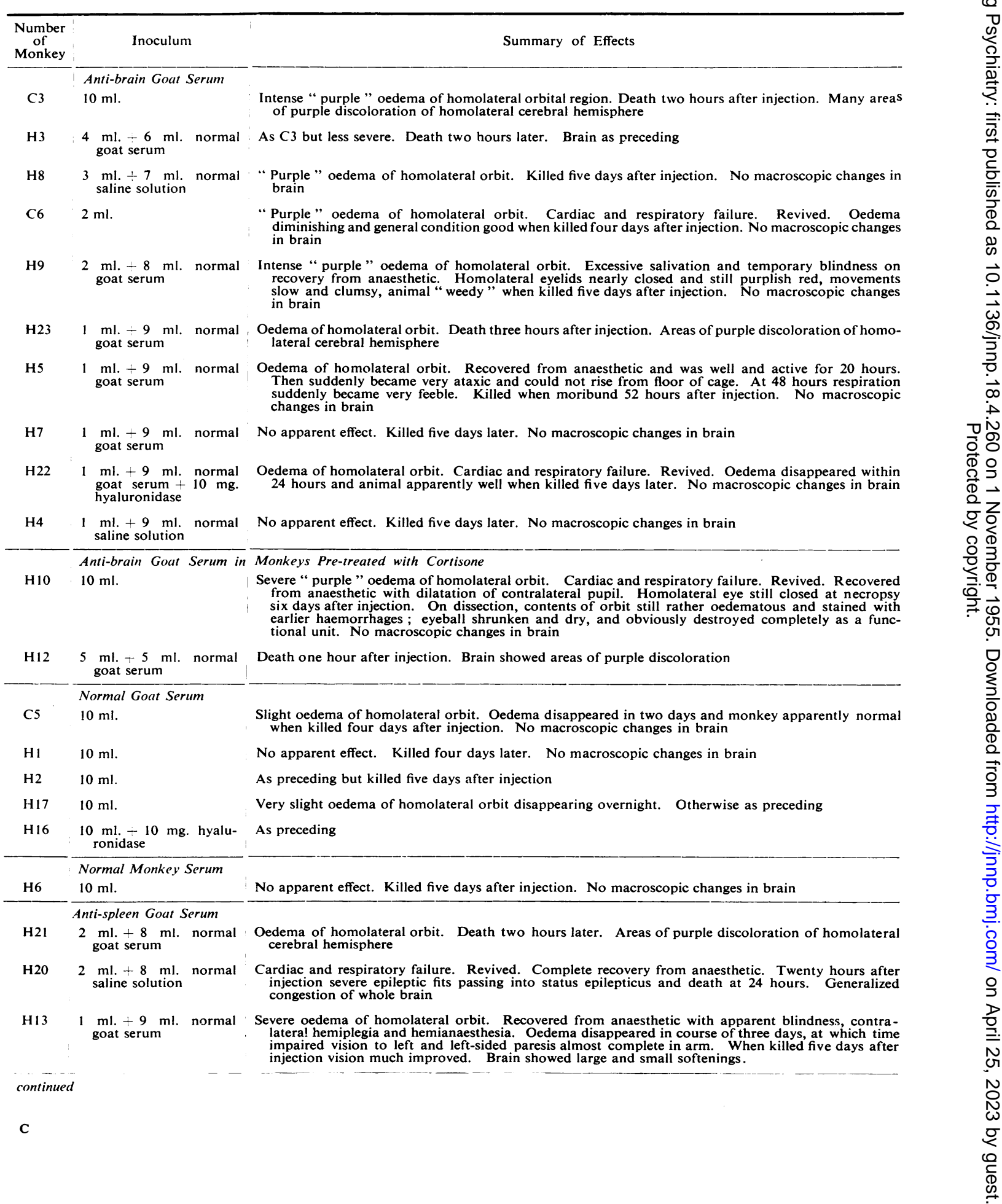




\begin{tabular}{|c|c|c|}
\hline $\begin{array}{l}\text { Number } \\
\text { of } \\
\text { Monkey }\end{array}$ & Inoculum & Summary of Effects \\
\hline H19 & $\begin{array}{l}\text { Anti-spleen Goat Serum } \\
0.7 \mathrm{ml} .+10.3 \mathrm{ml} \text {. nor- } \\
\text { mal goat serum }\end{array}$ & $\begin{array}{l}\text { Oedema of homolateral orbit. Cardiac and respiratory failure. Revived. On recovery from anaes- } \\
\text { thesia, contralateral pupil greatly dilated and vision defective to this side. Oedema disappeared } \\
\text { within } 72 \text { hours, but visual changes persisted until necropsy on fifth day. No macroscopic changes } \\
\text { in brain }\end{array}$ \\
\hline H18 & $\begin{array}{l}0.7 \mathrm{ml} .+10 \cdot 3 \mathrm{ml} \text {. nor- } \\
\text { mal goat serum }+3 \\
\text { mg. hyaluronidase }\end{array}$ & $\begin{array}{l}\text { Severe oedema of homolateral orbit. Cardiac and respiratory failure. Revived. On recovery from } \\
\text { anaesthetic animal blind, left eye deviated to contralateral side (right eye not visible), contralateral } \\
\text { hemiplegia and hemianaesthesia. Oedema, blindness, and deviation of eye disappeared in } 48 \text { hours. } \\
\text { Generalized tremor on movement and hemiparesis persisting at necropsy five days after injection. } \\
\text { Brain showed small and large softenings. }\end{array}$ \\
\hline H11 & $\begin{array}{l}\text { Anti-spleen Goat Serum in } \\
10 \mathrm{ml} \text {. }\end{array}$ & $\begin{array}{l}\text { Monkeys Pre-treated with Cortisone } \\
\text { Reddening but no oedema of homolateral orbit. Cardiac and respiratory failure. Revived. No } \\
\text { recovery of consciousness. Death seven hours after injection. Areas of purple discoloration of } \\
\text { homolateral cerebral hemisphere }\end{array}$ \\
\hline H14 & $\begin{array}{l}3 \mathrm{ml} .+7 \mathrm{ml} \text {. normal } \\
\text { goat serum }\end{array}$ & Oedema of homolateral orbit. Death one hour after injection. Brain as preceding \\
\hline
\end{tabular}

lateral eye, but once from the contralateral and once from both.

Over a period extending from within a few minutes to several hours after the operation, injected monkeys were very prone to sudden cardiac and respiratory failure and death. In several instances in which we countered impending collapse with intravenous or intracardiac adrenalin coupled with artificial respiration, we succeeded in preventing apparently certain death; on other occasions the treatment was of no avail.

On the day after injection of serum, several animals receiving small doses appeared normal. One (H9), however, which had been temporarily blind and had salivated vigorously on coming round from the anaesthetic, ate little food for 48 hours and remained slow and clumsy in all its movements until necropsy on the fifth day. A second (H5), apparently quite well until 20 hours after injection, then suddenly developed progressive nervous symptoms and was moribund at 52 hours. It is worth recalling that we have described similarly delayed and progressive symptoms as a sequel to chemical anoxia (Hurst, 1940, 1942).

Animals dying within an hour or two all exhibited areas of reddish or purple discoloration within the territories of the anterior and middle cerebral arteries of the homolateral cerebral hemisphere. On section the grey matter was intensely congested, the white matter less obviously so, though to some extent the congestion here was probably merely obscured by the whiteness of the areas. Animals killed two to six days after injection showed no certain macroscopic changes in the brain.

In one monkey, the addition of hyaluronidase to the serum in the hope of enhancing capillary permeability did not apparently increase the severity of the effects. In two monkeys pre-treatment with
$10 \mathrm{mg}$. $/ \mathrm{kg}$. cortisone 24 and two hours before operation may have modified slightly the reaction, but certainly did not prevent it. We received the impression that the effects of a given dose of serum diluted with physiological saline were less severe than those of an equivalent dose diluted with normal goat serum.

Pathological Examination.-Fig. 1 depicts the ̊ macroscopic appearances in the brain of $\mathrm{C} 3$ which died within two hours of the injection of serum. Intense purple discoloration, accompanied by considerable swelling, affected part of the basal surface of the homolateral frontal lobe and the temporal pole, extended on to the lateral surface where it missed the pre- and post-Rolandic convolutions, and reached the vertex in the hinder parietal area. This general distribution appeared to be fairly constant and corresponded with that of microscopical changes in the monkeys which survived. Much of the homolateral brain and the whole of the other side were free from congestion.

Animals killed after four to six days did not show clear macroscopic abnormality.

Microscopical examination in animals dying within one to three hours $(\mathrm{C} 3, \mathrm{H} 3,12,23)$ showed every vessel, large and small, in the affected areas to be stuffed with erythrocytes (Fig. 2). In the arteries and arterioles for the most part these appeared as discrete units, but in the more severely affected regions those in the capillaries tended to lose their outlines and fuse into rather granular plugs which still preserved the usual staining properties of red blood cells. Lying between the adventitia and the nervous substance outside many of the arteries and venules was a hyaline coagulum which stained metachromatically with toluidin blue. Occasional capillary or ball or ring haemorrhages were seen, 


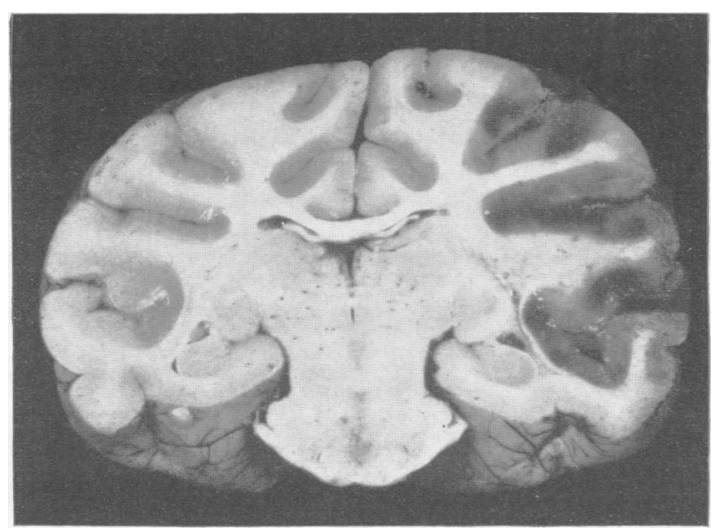

FIG. 1.-Intense congestion of some cortical areas two hours after intra-arterial injection of neurotoxic goat serum.

but were not a conspicuous feature. Even at two hours after injection the oligodendroglial cells in affected areas already exhibited pyknosis of the nuclei and acute swelling of the cytoplasm, and the nerve cells vacuolation of the cytoplasm and pericellular oedema.

Despite the absence of macroscopic alterations, several of the monkeys surviving longer showed lesions in the general areas outlined above.

In $\mathrm{H} 5$ and $\mathrm{H} 9$ these were numerous and often severe, particularly in the cortex of the gyri temporalis superior and inferior at the temporal pole and on the anterior part of the lateral surface of the temporal lobe ; further back in these gyri lesions more characteristically involved the white matter rather than the cortex and also affected the gyrus angularis, and in minor degree the gyrus centralis posterior, both in the present monkeys and in those to be described in a later section. Smaller areas of less severe damage occurred in adjacent parts of the cortex, in the nucleus amygdalae, in parts of the caudate and lenticular nuclei, in the wall of the third ventricle and subthalamic nuclei, and in the optic tract. The more severe lesions consisted in complete necrosis of the cerebral cortex over areas varying from pinheads to those of $0.5 \mathrm{~cm}$. or more in diameter. In these areas many capillaries were still blocked by the granular plugs derived from altered red blood corpuscles, but ordinary thrombi were not seen; the capillary endothelial cells in many cases were necrotic. In the case of longer duration (H9), the less severe lesions in the grey matter were characterized by moderate to severe vacuolation of nerve cells, sponginess of the ground substance, ballooning and fragmentation of myelin sheaths, formation of plasma-rich and often binucleate glial cells, early reaction on the part of the microglia, and swelling and proliferative activity of vascular adventitial or endothelial cells. The hyaline coagulum outside certain vessels had been resolved into globules which were undergoing phagocytosis. The white matter of the gyrus temporalis superior in its middle and posterior parts showed considerable pallor and swelling, as compared with the corresponding area of the opposite side ; the arcuate fibres were less affected than the rest (Fig. 3). The myelin sheaths were separated by oedema and many were ballooned or fragmenting, while the neuroglial cells were enlarged and often binucleate and the microglia in an early stage of reaction. No fat was detected. This picture is very similar to that described by Greenfield (1939) in cerebral oedema. In the central parts of the optic tracts, small foci of ballooning or breakdown of myelin and of complete or almost complete softening often co-existed in the same section. In H5 similar lesions were at a less advanced stage of evolution. It is to be noted that in regions of damage short of complete necrosis the vessels were all normally patent; if therefore they had suffered obstruction from the erythrocytic plugs mentioned

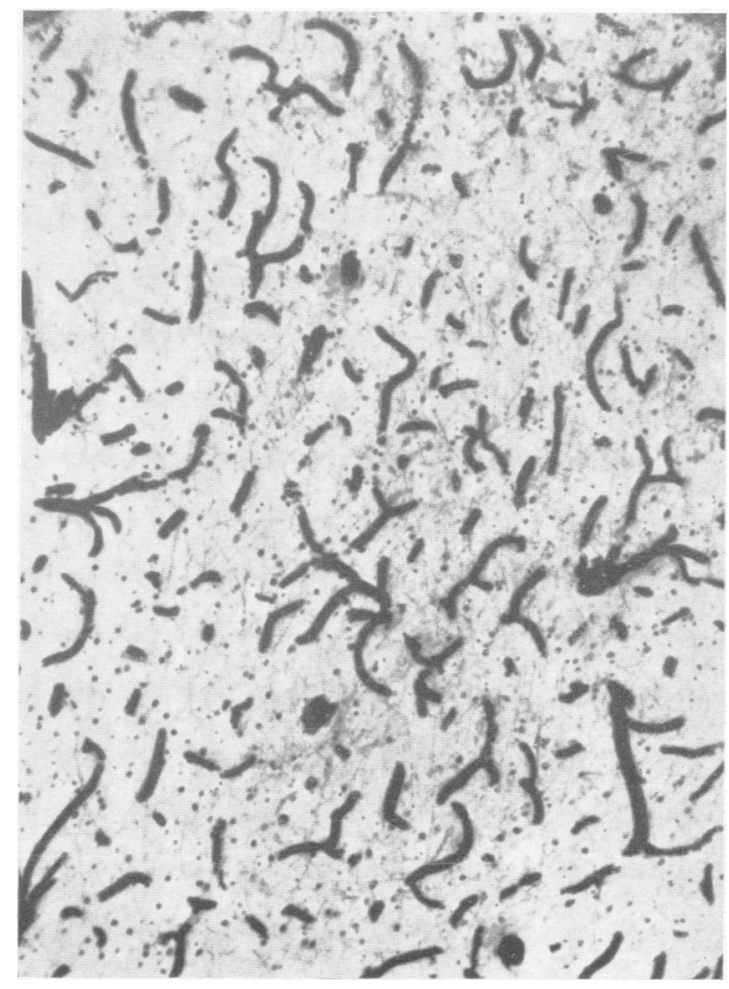

FIG. 2.-The microscopical picture corresponding to Fig. 1 as seen in a seztion stained by Weil's stain for myelin. 


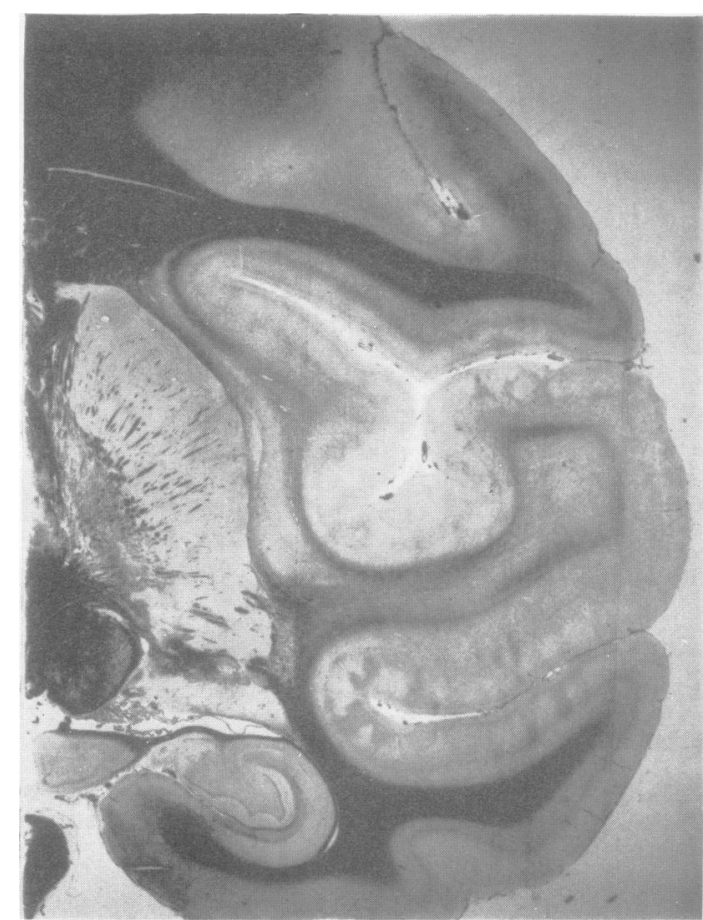

FIG. 3.-Oedema and demyelination in the white matter of the gyrus temporalis superior five days after neurotoxic serum. Lesions can be discerned also in the adjacent cortex and in the optic tract. Weil's stain.

above, these plugs had later been dislodged and the circulation re-established.

Similar but much less extensive and severe lesions obtained in $\mathrm{C} 6$ and $\mathrm{H} 10$, while only the optic tract was involved in $\mathbf{H 8}$. No nervous lesions were detected in $\mathrm{H} 4,7$, and 22.

Although as stated previously, in the hours following the injection of serum, the "purple" oedema of the eyelids seemed to be a general indicator of the state of affairs within the cranium, the course of events in the brain and the eye did not necessarily run completely parallel. Thus H5 with severe nervous lesions suffered but moderate oedema of the eye, while $\mathrm{H} 10$ with comparatively mild cerebral changes showed extremely severe orbital destruction. In $\mathrm{H} 10$ the optic nerve was completely necrotic, as was the retina; the former was devoid of any recognizable cellular remains, but in the latter large numbers of invading polymorphonuclears, also necrotic, could be discerned. Other nerves lying within the highly oedematous orbit were less severely damaged or were normal ; there was, however, extensive necrosis of fat and muscle, with lively proliferation of sarcolemmal nuclei and cells of the vascular adventitia in some places and no cellular reaction whatsoever in others. Although a minority of vessels appeared to be plugged with agglutinated red cells many seemed to be patent. Lumsden (1950) has described necrosis of the globe of the eye following the introduction of minute emboli into the carotid artery.

\section{Effects of Injecting Normal Sera}

Five monkeys, receiving very large doses of normal goat serum, with or without hyaluronidase, either suffered no obvious ill-effects (H1 and 2) or, after slight retching during the injection, developed very transient and slight orbital oedema which was never "purple". At necropsy four to five days later the brains appeared normal.

One monkey receiving a large dose of normal monkey serum manifested no ill-effects.

Pathological Examination.-No microscopic changes were found in the brains of these animals.

\section{Effects of Injecting Anti-spleen Serum}

At any given dose this serum produced more severe effects than did the neurotoxic serum.

Oedema of the orbit, though not "purple ", was often severe. Two monkeys surviving the immediate effects of the injection recovered from the anaesthetic apparently blind. Residual symptoms included tremor on movement, and contralateral hemianopia, hemiparesis, and hemianaesthesia. One animal apparently well for 20 hours then developed convulsions and died at 24 hours in status epilepticus.

At necropsy on animals dying acutely, areas of purple discoloration were seen in the homolateral cerebral hemisphere. The brain of the monkey dying of convulsions at 24 hours was generally congested. At necropsy on cases surviving five days, two of three monkeys showed macroscopic softenings in the homolateral cerebrum; these varied from purple pin-heads in parts of the cortex to extensive softenings (up to $1 \mathrm{~cm} . \times 0.75 \mathrm{~cm}$.) in the cortex, basal nuclei, or white matter. Haemorrhages were more frequent than with the neurotoxic serum.

Hyaluronidase did not appear to augment the damage, nor pre-treatment with cortisone appreciably to mitigate it.

Pathological Examination.-The microscopical changes in monkeys which succumbed within a few hours were indistinguishable from those following administration of neurotoxic serum. At seven hours (H11), in addition to the changes already mentioned, the capillary endothelial cells were pyknotic or apparently missing from the most severely affected areas. 
In animals surviving longer, lesions were larger, more severe, and occurred over a wider area than with neurotoxic serum.

$\mathrm{H} 20$, dying in status epilepticus after 24 hours, showed generalized congestion of the brain with more intense congestion, capillary plugging, and incipient necrosis in the homolateral superior temporal gyrus, lenticular nucleus, etc.

H13, 18, and 19, killed on the fifth day, exhibited very severe and widespread softenings with more numerous haemorrhages than obtained in the neurotoxic group. All the areas previously mentioned as affected were more severely involved and, in addition, the internal capsule, anterior commissure, optic thalamus, centrum semi-ovale, and some areas of the cortex not previously observed to be damaged, e.g., the gyrus hippocampi, were here affected. The internal capsule particularly was the site of multiple severe injuries in $\mathrm{H} 13$ and 18, a fact reflected in the symptomatology during life. In parts of the white matter undergoing rapid and complete necrosis we made an observation previously recorded in chemical necrosis of the white matter (Hurst, 1940, 1942), namely that in these severely stricken areas the myelin sheaths quickly lose their ability to stain by Weil's method while retaining their normal form and contour, whereas in areas of partial damage the tinctorial properties are normal but the outline of the sheaths is grossly altered by beading, ballooning, etc. The optic tracts and nerves were severely damaged in all, but the retina was destroyed only in $\mathrm{H} 19$ and in none of the monkeys were the orbital changes nearly as severe as in H10.

The lesions caused by the anti-spleen serum were thus quantitatively more severe than those produced by anti-brain serum, but, using the intra-arterial route of injection, we were unable to detect any qualitative difference in the two series.

\section{Comment}

The injection into the cerebral circulation of anti-sera to either brain or spleen apparently damages the walls of the capillaries and may lead to intense congestion in territories (including the orbit) supplied by the arteries concerned. Where damage is most severe, we find the small vessels lastingly obstructed with granular plugs consisting of altered red blood cells, and full necrosis occurs in the surrounding nervous and orbital tissues.
Where damage is less severe, we may suppose either that obstruction is temporary and that the circulation is later restored with only moderate injury to the nervous tissues, or that these suffer as a result of altered permeability or other change in the vessels ; in either case the ensuing lesions fall short of full necrosis. The former explanation of the genesis of this partial damage seems the more convincing since the more toxic anti-spleen serum, though certainly coursing through the same vascular channels as the anti-brain serum, produced far more widespread softenings with many more lastingly blocked capillaries and fewer areas of partial damage. However this may be, the lesions bear an obviously vascular stamp, and those evoked by neurotoxic serum do not appear different, apart from their lesser severity, from those arising from the action of anti-spleen serum. Thus we failed to obtain evidence that a neurotoxic serum given by the intra-arterial route is capable of direct assault on the nervous tissues, or in more general terms that an antigen on one side of the blood-brain barrier is capable of reacting with antibodies on the other. Without making any assumption as to the nature and relative sites of hypothetical antigen and antibody in some acute nervous diseases of obscure origin in man, we had hoped to produce in the experimental animal a condition resembling (say) acute haemorrhagic leucoencephalitis. We were disappointed, and from this point of view the observations have only a negative value.

\section{Summary}

Anti-brain and anti-spleen goat sera injected into the common carotid artery of monkeys, after ligation of branches other than the internal carotid, produce serious lesions in the vascular territories concerned. Damage to the vessel walls results in intense congestion, and the capillaries may become lastingly obstructed by plugs of altered red blood cells. Ischaemic changes follow in the nervous tissues. The anti-spleen serum was the more noxious.

\section{REFERENCES}

Greenfield, J. G. (1939). Brain, 62, 129

Hurst, E. W. (1940). Aust. J. exp. Biol. med. Sci., 18, 201. - (1942). Ibid., 20, 297.

1955); Journal of Neurology, Neurosurgery and Psychiatry, $18,174$.

Lumsden, C. E. (1950). Ibid., 13, 1. 